\title{
EXPERIMENTAL MODELING APPROACH FOR DETERMINING THE MOISTURE DAMPING EXPONENT OF A BLUETOOTH LOW ENERGY SIGNAL IN MOIST BUILDING MATERIAL
}

\author{
Laura Moldenhauer ${ }^{1}$, Rosemarie Helmerich ${ }^{2}$, Enrico Köppe ${ }^{3}$, Jochen Wittmann ${ }^{4}$ \\ 1 Federal Institute for Materials Research and Testing (BAM), Unter den Eichen 87, 12205 Berlin, \\ Germany. E-Mail: laura.moldenhauer@bam.de; rosemarie.helmerich@bam.de; \\ 2 University of Applied Sciences, HTW, Wilheminenhoffstr., 12587 Berlin, Germany.
}

\section{Introduction}

The presented development of a damping model is a research component of an experimental feasibility study about moisture in building materials measured with Bluetooth ${ }^{\circledR}$ Low Energy (BLE) signals [1]. This study may be part of a structural health monitoring [2] aiming on early damage detection in the built infrastructure and is increasingly focusing on wireless sensor network technology [3]. It is investigated, how the Received Signal Strength Indicator (RSSI) of a BLE signal, transmitted from the BLE-module embedded in building materials with changing moisture content is damped. The BLE-module communicates with a mobile Smart Device as tablet or mobile phone via 2.45 GHz-ISMfrequency band where water dipoles start to oscillate. If the BLE-signal transfers through a moist material, the moisture content influences the RSS-Indicator.

The damping model demonstrates this damping effect on RSSI by the abstraction of the reality observed in a real system. Through the reproduction of the active relationships, the system behavior is decomposed in such a way that a separate consideration of the required system parameters is possible. The aim of the modelling is the derivation of a damping equation for the formal model to determine the moisture damping exponent to find a correlation.

\section{Model development}

The assumption is that the signal damping corresponds to the power transmission balance. This balance is the sum of the three damping components; Environmental damping, material damping (environment + material) and moisture damping (environment + material + moisture). Therefore, a model for these damping variables is required. The development of the formal model refers to the Log-Distance Path Loss Model (FSPL) Eq. (1) [3].

$\boldsymbol{F S P L}=10 * \log _{10}\left(\frac{4 \pi \mathbf{r} * \mathbf{f}}{\mathbf{c}}\right)^{2}$

$\mathbf{4 \pi r}=$ Area of a circle from a antenna

$\mathbf{f}=$ is the signal frequency (in Hertz),

$\mathbf{c}=$ is the velocity of light in a vacuum

This model, known from electrical engineering, is not applicable. It applies only in a vacuum and does not consider the influence of the distance. Provided the distance high $-n$ (negative path loss exponent) is proportional to the received power, the equation can be extended to the Log-Distance Path Loss Modell Eq. (2).

$\mathbf{R S S I}_{\mathbf{d}}=-(10 * \mathbf{n}) \log _{10}\left(\frac{\mathbf{d}}{\mathbf{d}_{\mathbf{0}}}\right)-\mathbf{R S S I}_{\mathbf{d}_{\mathbf{0}}}$

$\mathbf{n}=$ Path loss exponent (The exponent $\mathbf{n}$ increases with increasing damping)

$\mathbf{d}=$ Distance between transmitter and receiver

$\mathbf{d}_{0}=$ Distance between transmitter and receiver (rm)

RSSI $_{\mathrm{d}}=$ Received signal strength indicator in $\mathrm{dBm}$

RSSI $_{\mathrm{d} 0}=$ Received signal strength indicator in $\mathrm{dBm}(\mathrm{rm})$

The Log-Distance Path Loss Model is applied by switching on $\mathbf{n}$ for determining the damping components Eq. (3).

RSSI $_{\text {Environment }}$

$=-\left(10 * \mathbf{n}_{\text {Environment }}\right) * \log _{10}\left(\frac{\mathbf{d}}{\mathbf{d}_{\mathbf{0}}}\right)-\mathbf{R S S I}_{\mathbf{d}_{\mathbf{0}}}$ (3)

At first, the environmental damping was considered. After insertion and conversion, the equation (4) is obtained. 


$$
\begin{aligned}
& \mathbf{n}_{\text {Environment }} \\
& =\frac{\text { RSSI }_{\text {Environment d }_{\mathbf{d}}+\mathbf{R S S I}_{\mathbf{d}_{\mathbf{0}}}}}{-10 * \log _{10}\left(\frac{\mathbf{d}}{\mathbf{d}_{\mathbf{0}}}\right)}
\end{aligned}
$$

If the material damping is considered, the signal damping is the sum of environment and material damping, as shown in equation (5).

$$
\begin{aligned}
& =\frac{\mathbf{R S S I}_{\text {Material d }}+\mathbf{R S S I}_{\text {Material } \mathbf{d}_{\mathbf{0}}}}{-10 * \log _{10}\left(\frac{\mathbf{d}}{\mathbf{d}_{\mathbf{0}}}\right)} \\
& \quad-\mathbf{n}_{\text {Environment }}
\end{aligned}
$$

The same damping is considered as the result for moisture. Eq. (6).

$$
\begin{aligned}
& =\frac{\mathbf{R S S I}_{\text {Moisture d }}+\mathbf{R S S I}_{\text {Moisture d }_{\mathbf{0}}}}{-10 * \log _{10}\left(\frac{\mathbf{d}}{\mathbf{d}_{\mathbf{0}}}\right)} \\
& \quad-\mathbf{n}_{\text {Environment }}-\mathbf{n}_{\text {Material }}
\end{aligned}
$$

\section{Data validation and results}

The hypotheses for the damping models were validated with the analyzed system data. In the evaluation of the experimental system data we can establish a proportional relationship between the moisture content and the logarithm of the received strength. To do this, the model experiment was carried out without moisture. By transferring the experimental data to the formal model, the following values are obtained for the environment and the material. See Fig. 1.

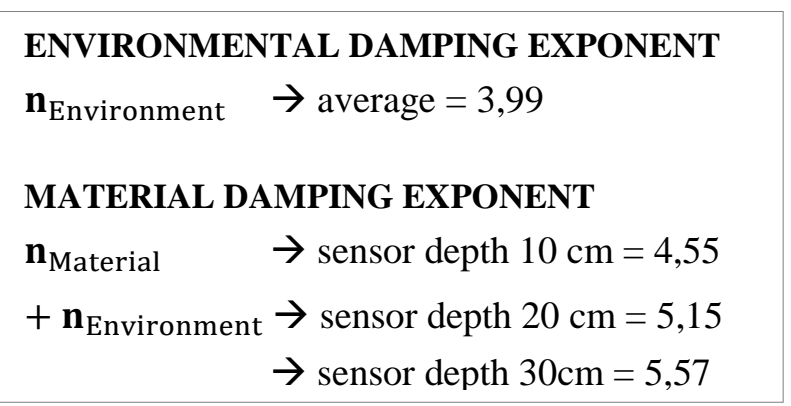

Fig 1: Results of the damping exponets

The evaluation of the system data and its application to the formal model has resulted in the environment damping exponent of 3.99. This damping exponent for the environment correlates with the expectations for shaded buildings. The material and moisture damping are determinable with the model and grow in exponential relation to the signal path in the material, see Fig 2.

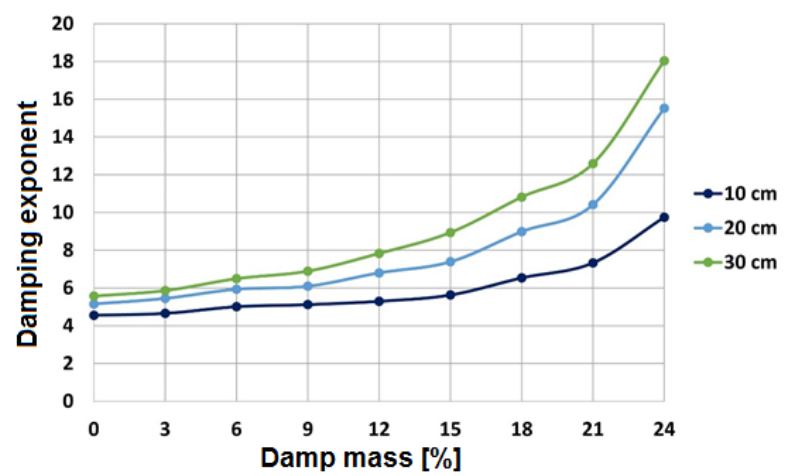

Fig 2:Damping exponents in moist building material in three different sensor dephts

The calculated damping exponents from the model of the investigated moisture content (in \%) show the dependence of the RSSI on the damp mass inside the building material. It can be observed, that the exponents of the damping grow continuously exponentially with the moisture content. said it can be concluded that water massively attenuates the BLE signal during data transmission. Based on the RSSI, it is possible to derive statements about the moisture content in the material. In accordance with the results of the model, the information value of moisture content decreases with the embedding depth of the sensor.

\section{Acknowledgements}

The experimental work was carried out during my Bachelor thesis. The contribution to the project for BLE sensor development and application to construction monitoring funded during a German ZIM project, by the German Minister of Economy and Energy is kindly acknowledged.

\section{References}

[1] Moldenhauer, L., Investigation and visualization of the damping of Bluetooth Low Energy signals under the influence of damp mass in building materials, Bachelor thesis HTW, Berlin, 2016

[2] Köppe, E., Bartholmai, M., Long term monitoring and early damage detection in structures applying an energy wireless efficient sensor network Presentation TC Structural Health Monitoring, Airbus, Hamburg, 2008

[3] Helmerich, R., Nondestructive Evaluation and Structural Health Monitoring, Civil Structural 


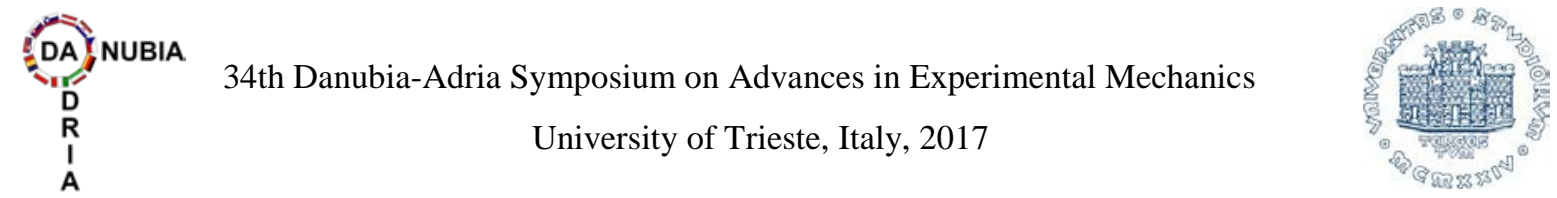

Health Monitoring Workshop, CSHM-5, Ube, Japan, 2008 